\title{
Spektrum Onkologie - wissenswert, kompakt, anregend
}

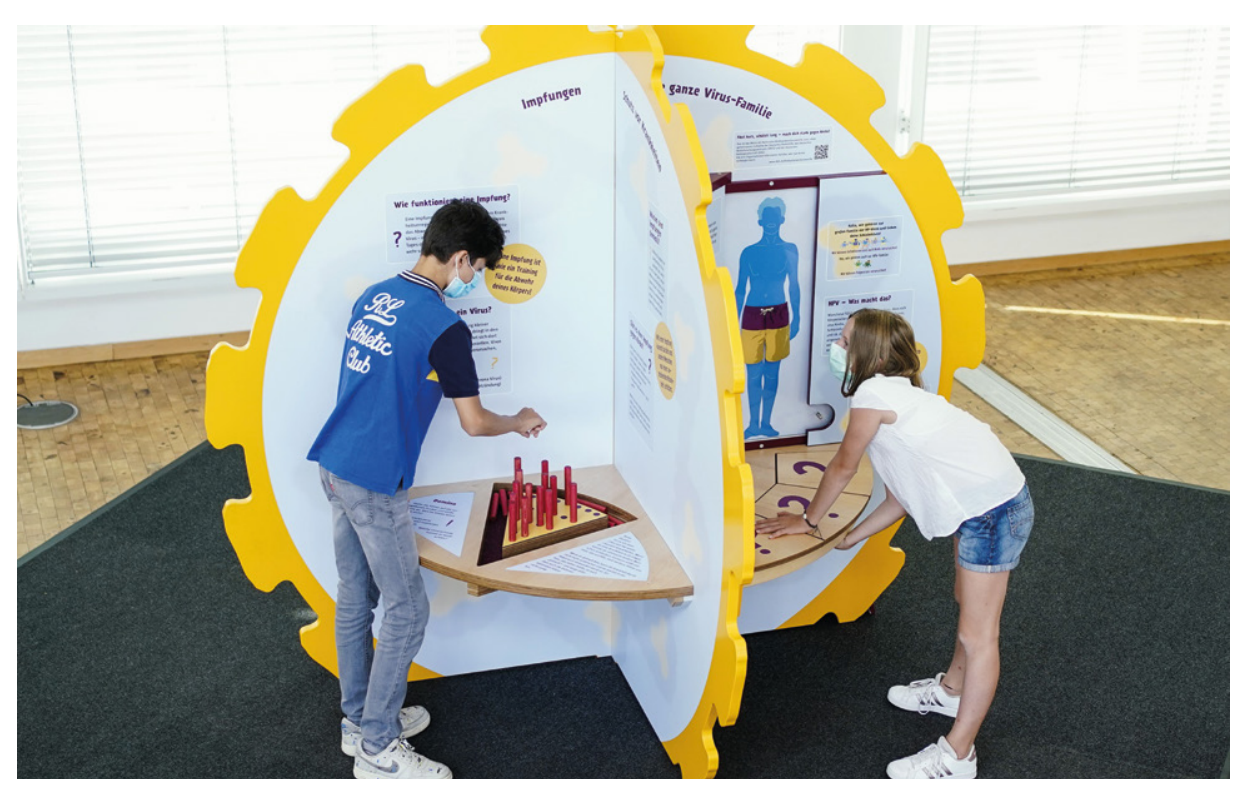

An der HPV-Mitmachstation erkunden Jugendliche spielerisch, wie eine HPV-Impfung vor bestimmten Krebsarten schützt. Foto: U. Anspach/DKFZ

\section{Nationales Centrum für Tumorerkrankungen Dresden (NCT/UCC) FOKUS}

\section{Riesiges Virus informiert spielerisch über den Schutz vor HPV-bedingten Krebserkrankungen}

Humane Papillomviren (HPV) sind in Deutschland jedes Jahr für rund 7700 Krebsneuerkrankungen verantwortlich. Eine rechtzeitig vorgenommene HPV-Impfung schützt hochwirksam vor den krebserregenden Virustypen, wird aber deutschlandweit noch viel zu selten in Anspruch genommen. Das Nationale Centrum für Tumorerkrankungen Dresden (NCT/UCC) lädt dazu ein, an einem großen Virus-Modell spielerisch zu erkunden, welche Folgen eine Infektion mit HP-Viren haben kann und wie eine HPV-Impfung vor bestimmten Krebsarten schützt. Die Mitmachstation mit Geschicklichkeits- und Tastspielen steht noch bis Ende November im Universitäts Kinder-Frauenzentrum des Universitätsklinikums Carl Gustav Carus Dresden. Entwickelt wurde sie anlässlich der Nationalen Krebspräventionswoche vom Deutschen Krebsforschungszentrum, von der Deutschen Krebshilfe und der Deutschen Krebsgesellschaft.
«Die HPV-Impfung ist ein Quantensprung zur Vorbeugung HPV-bedingter Krebserkrankungen. Leider ist die Impfquote in Deutschland viel zu niedrig für einen flächendeckenden HPV-Schutz. Mit der Mitmachstation möchten wir Eltern und Kinder spielerisch über die Vorteile der HPV-Impfung informieren», sagt Prof. Pauline Wimberger, Direktorin der Klinik für Frauenheilkunde und Geburtshilfe des Universitätsklinikums Carl Gustav Carus Dresden und Mitglied im erweiterten Direktorium des Nationalen Centrums für Tumorerkrankungen Dresden (NCT/UCC).

Nachdem 1971 in Deutschland das Angebot zur Früherkennung von Gebärmutterhalskrebs durch einen jährlichen Zellabstrich vom Gebärmutterhals (PAP-Abstrich) eingeführt wurde, sanken die Erkrankungsraten um mehr als die Hälfte. In den letzten 15 Jahren ist die Zahl der jährlichen Neuerkrankungen hingegen stabil. «Durch die
Impfung haben wir nun die Chance, den Gebärmutterhalskrebs weitgehend auszurotten. Es ist wichtig, dass wir diese Chance nutzen», betont Prof. Wimberger.

Auch in der Therapie des Gebärmutterhalskrebses gibt es Fortschritte. So können Patientinnen am NCT/UCC in der Universitätsfrauenklinik mit einer besonderen nervenschonenden Methode - der so genannten totalen mesometrialen Resektion (TMMR) - operiert werden. Die Methode wurde von Prof. Michael Höckel an der Universität Leipzig entwickelt und wird deutschlandweit bislang nur an wenigen Zentren angeboten. Ein Durchbruch kündigt sich aktuell in der Standardtherapie für bestimmte metastasierte Gebärmutterhalskrebserkrankungen an: Eine Immuntherapie mit dem Checkpoint-Inhibitor Pembrolizumab soll künftig zusätzlich zur Chemotherapie zum Einsatz kommen. 


\section{Auszeichnung für herausragende Krebsforscher}

Prof. Dr. Tobias Bald, Universitätsmedizin Bonn, und Prof. Dr. Sebastian Kobold vom Klinikum der Ludwig-Maximilians-Universität München erhielten Anfang November 2021 den LisecArtz-Preis. Die Bonner Universitätsstiftung und die Medizinische Fakultät der Universität Bonn schreiben den mit 10000 Euro dotierten Preis deutschlandweit öffentlich aus. Damit ehrt die Stiftung herausragende Nachwuchsforschende in der Krebsforschung.

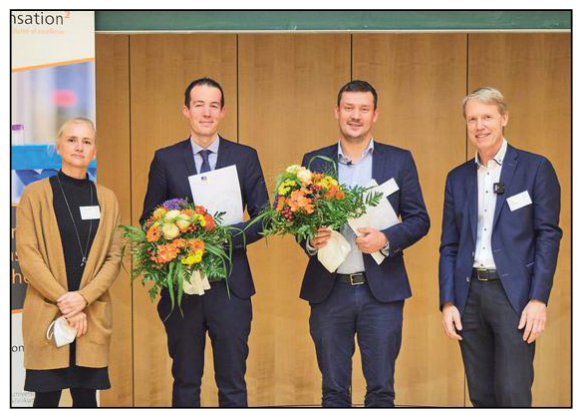

Prof. Dr. Dagmar Wachten (Vorstand der Bonner Universitätsstiftung), Prof. Dr. Sebastian Kobold, Prof. Dr. Tobias Bald und Prof. Dr. Gunther Hartmann (Prodekan für Forschung der Medizinischen Fakultät). Foto: Volker Lannert/Uni Bonn
Tobias Bald ist Professor für Tumor-Immunbiologie des Exzellenzclusters ImmunoSensation2 sowie Mitglied im Transdisziplinären Forschungsbereich (TRA) der Universität Bonn «Leben und Gesundheit». Er forscht zum T-Zellen aktivierenden Rezeptor CD226. Neben Infektionen schützt das Immunsystem auch vor Krebserkankungen, in dem es bösartig veränderte Zellen frühzeitig erkennt und zerstört. Tobias Bald versucht mit seinem Team seit Jahren, die Interaktionen zwischen Krebszellen und Immunzellen besser zu verstehen. Kürzlich zeigte er zum Beispiel, dass Krebszellen das Immunsystem auf bisher unbekannte Weise schwächen können. Immunzellen brauchen im Kampf gegen Krebszellen ständig positive Signale - eine Art Motivation zum Weitermachen. Dies haben Krebszellen erkannt und entziehen den Immunzellen die positiven Signale. Die Immunzellen werden dadurch so geschwächt, dass die Krebszellen nicht mehr zerstört werden können. Das Team arbeitet mit Hochdruck daran, dieses neue Wissen zu nutzen, um die Krebsimmuntherapie deutlich zu verbessern.

\section{Pionierarbeit für die Immuntherapie}

Die Arbeitsgruppe um Professor Kobold befasst sich mit der Entwicklung neuer Behandlungsmöglichkeiten gegen Krebserkrankungen. Die Forschenden machen das körpereigene Immunsystem durch synthetische Eiweiße scharf. Dadurch erlangen die Immunzellen die Fähigkeit, Krebszellen zu erkennen und zu zerstören. Gleichzeitig ermöglicht die Methode den Zellen des Abwehrsystems, unbeschadet und gezielt in bösartige Gewebe zu wandern. Hier konnte die Arbeitsgruppe Pionierarbeit leisten, weil es über bereits existierende Immuntherapien zur Behandlung fortgeschrittener Blutkrebsarten hinaus Bedarf etwa für Brust-, Lungen- oder Darmkrebs gibt. An diesen Erkrankungen mit hohem medizinischen Bedarf forscht Professor Kobold schwerpunktmäßig. Aktuell arbeitet sein Team an der weiteren Entwicklung in Richtung klinischer Studien.

\section{Martin-Luther-Universität Halle-Wittenberg}

\section{Neues Graduiertenkolleg der Universitätsmedizin Halle erforscht Entstehung von Bauchspeicheldrüsenkrebs}

Ein neues Graduiertenkolleg (GRK) der Deutschen Forschungsgemeinschaft (DFG) an der Universitätsmedizin Halle erforscht die Entstehung von Bauchspeicheldrüsenkrebs, dem sogenannten Pankreaskarzinom. Bauchspeicheldrüsenkrebs ist nach wie vor eine der Krebserkrankungen mit der schlechtesten Prognose, unter anderem auch, weil die Tumoren oft erst sehr spät entdeckt werden. Chronische Entzündungen sind für Bauchspeicheldrüsenkrebs wie auch viele andere Tumorerkrankungen ein Risikofaktor. Die eigentliche Entstehungsursache der Karzinome und welche Faktoren dafür verantwortlich sind, ist nach wie vor nicht gut erforscht.

Das Graduiertenkolleg «Entzündliche Einflüsse als Modulatoren der frühen Pankreaskarzinogenese (InCuPanC)» will daher den Einfluss verschiedener entzündlicher Signale in der frühen Phase der Krebsentstehung systematisch entschlüsseln. Das GRK wird von der DFG mit bis zu fünf Millionen Euro über einen Zeitraum von viereinhalb Jahren gefördert.

«Ziel unseres Graduiertenkollegs ist es, neue Ansätze zur Früherkennung von Bauchspeicheldrüsenkrebs zu finden, denn das Wissen über die molekularen Mechanismen der entzündungs-assoziierten frühen Tumorentwicklung im Pankreas immer noch sehr begrenzt. Unser GRK will den Einfluss verschiedener entzündlicher Signale, die von genetischen, metabolischen oder äußeren Auslösern induziert werden, speziell auf die Transition von präinvasiven Vorläuferläsionen zu invasiven Tumoren im Rahmen der frühen Karzinogenese im Pankreas systematisch zu entschlüsseln», sagt Prof. Dr. Patrick Michl. Der Direktor der Universitätsklinik und Poliklinik für Innere Medizin I (Gastroenterologie, Pneumologie) der Universitätsmedizin Halle ist Sprecher des neuen GRK. Beteiligt daran sind in insgesamt 13 Projekten Wissenschaftlerinnen und Wissenschaftler der Klinik für Innere Medizin I, der Klinik für Innere
Medizin IV (Hämatologie, Onkologie), der Klinik für Viszerale, Gefäß- und Endokrine Chirurgie und der Institute für Pathologie, Molekulare Medizin und Anatomie und Zellbiologie der Universitätsmedizin Halle sowie vom Institut für Pharmazie der Martin-Luther-Universität Halle-Wittenberg.

Innerhalb des GRK finden verschiedenste Methoden der Grundlagenforschung und der translationalen Pankreasforschung einschließlich innovativer in vivo-Modelle, Proteom-Analysen und CRISPR-basierter Screens Anwendung, die den künftigen Doktorandinnen und Doktoranden Forschung auf modernstem Niveau ermöglicht.

Das Graduiertenkolleg wird zunächst bis Sommer 2026 gefördert. Eine Verlängerung der Förderung ist nach positiver Evaluierung möglich. Graduiertenkollegs sind ein Instrument zur Förderung des wissenschaftlichen Nachwuchses an Hochschulen und werden von der DFG bis zu neun Jahre gefördert. 


\section{Premiere: Protonentherapie gegen Lungenkrebs}

Am Paul Scherrer Institut PSI, Villigen/Schweiz, wurde Anfang November 2021 eine an Lungenkrebs erkrankte 60-jährige Patientin mit Protonen, also positiv geladenen Teilchen, bestrahlt. Es ist das erste Mal in der Schweiz, dass diese Art der Bestrahlung zur Krebsbehandlung an der Lunge eingesetzt wird. Die siebenwöchige Therapie erfolgt im Rahmen einer internationalen Studie, an der das PSI im Verbund mit dem Kantonsspital Aarau teilnimmt.

«Im Rahmen einer internationalen Phase-III-Studie haben wir heute die erste Patientin mit Lungenkrebs, genauer einem nicht-kleinzelligen Bronchialkarzinom, mittels Protonentherapie behandelt», sagt Damien Weber, der Chefarzt und Leiter des Protonentherapiezentrums am PSI. «Die Patientin hat einen Tumor im fortgeschrittenen Stadium, der nicht operiert werden konnte.» Die Studie wird von der amerikanischen Organisation für klinische Studien NRG Oncology geleitet. Das PSI nimmt gemeinsam mit dem Radio-Onkologie-Zentrum der Kantonsspitäler Aarau (KSA) und Baden (KSB) an der Studieteil-als einzige Einrichtungen ausserhalb

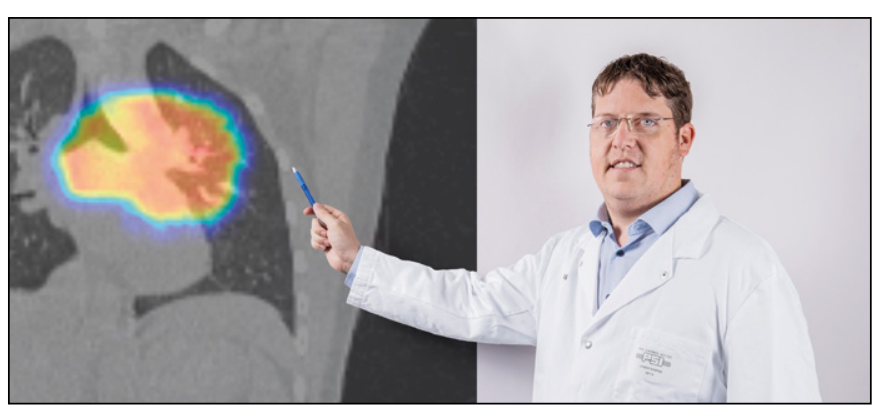

Dominic Leiser, der behandelnde Radioonkologe der ersten Patientin, die am PSI im Rahmen der Studie betreut wird. Er zeigt auf die computertomografische Aufnahme eines Lungenkarzinoms. Das farblich hervorgehobene Gebiet wird vom Protonenstrahl abgetastet. Foto: PSI

der USA. Die Studie vergleicht den Behandlungserfolg von herkömmlicher Strahlentherapie mit der von Protonentherapie beim nichtkleinzelligen Bronchialkarzinom - der häufigsten Form von Lungenkrebs - im fortgeschrittenen, inoperablen Stadium.

«Dass unser Radio-Onkologie-Zentrum und das PSI an der Studie mitwirken dürfen, ist nur aufgrund der langjährigen Expertise unserer beiden Einrichtungen auf dem Gebiet der Strahlentherapie und unserer Mitgliedschaft bei NRG Oncology möglich», sagt Oliver Riesterer, der Chefarzt des Radio-Onkologie-Zentrums Aarau und Baden. Die Zuteilung der Patienten erfolgt per Losverfahren: Einige werden mit Protonen am PSI bestrahlt, andere wie bisher mit Röntgenstrahlen am Radio-Onkologie-Zentrum KSA-KSB.

Insgesamt sollen in die Studie 330 Patienten eingeschlossen werden, etwa zehn davon in der Schweiz. «Die Zusammenarbeit unserer beiden Institutionen ist ein Meilenstein für die Krebspatienten im Kanton Aargau», sagt Weber. Auch in anderen Projekten wollen die beiden Einrichtungen künftig enger zusammenarbeiten. Riesterer betont: «Unser Ziel ist es, Photonen- und Protonentherapie bestmöglich zum Wohle unserer Patienten einzusetzen.»

\section{Deutsche Krebsgesellschaft e. V.}

\section{S3-Leitlinie Speiseröhrenkrebs aktualisiert}

Das Leitlinienprogramm Onkologie hat unter Federführung der Deutschen Gesellschaft für Gastroenterologie, Verdauungs-und Stoffwechselkrankheiten (DGVS) e. V. die S3-Leitlinie zum Ösophaguskarzinom aktualisiert und neue Empfehlungen zur Diagnostik sowie zur kurativen und palliativen Therapie formuliert. Die Leitlinie soll dazu beitragen, für Betroffene mit Speiseröhrenkrebs eine angemessene und evidenzbasierteGesundheitsversorgungsicherzustellen. Die Diagnostik und Therapie des Speiseröhrenkrebses ist sehr komplex. Die Speiseröhre befindet sich nahe am Bronchialsystem und an der Lunge. Dies erfordert hohe technische Anforderungen beim operativen Eingriff und eine enge Zusammenarbeit verschiedener Fachdisziplinen. Zudem können neben der Operation weitere Therapiemöglichkeiten in Betracht kommen, wie etwa die Radiotherapie, die Chemotherapie sowie die Endoskopie.

Kleinere, auf die Schleimhaut begrenzte Tumoren, werden in der Regel endoskopisch entfernt. Sind die Tumoren bereits tiefer eingewachsen, kann in bestimmten Fällen eine Operation als kurative Therapie infrage kommen. In einer Ösophagektomie wird dabei die gesamte Speiseröhre entfernt. Die Ösophagektomie und die Rekonstruktion des Ösophagus sind komplexe Eingriffe und können entweder minimalinvasiv oder in Kombination mit offenen Verfahren durchgeführt werden. «Neue Daten zeigen, dass hierbei die minimalinvasive oder Hybrid-Technik gegenüber dem offenen Verfahren Vorteile hat. Patientinnen und Patienten haben beispielsweise weniger postoperative Komplikationen, eine verbesserte Lebensqualität und zudem eine verbesserte Prognose», so Professor Dr. Rainer Porschen vom Kreiskrankenhaus Osterholz. Er ist zusammen mit Professor Dr. Matthias Ebert von der Universitätsmedizin Mannheim, Koordinator der S3-Leitlinie. Beim Ösophaguskarzinom werden zwei Gruppen unterschieden: Das Plattenepithelkarzi- nom tritt in der Speiseröhre meist in Höhe des Hals- und Brustbereichs auf. Adenokarzinome entstehen in der Regel im unteren Speiseröhrenabschnitt, der in den Magen mündet. Der Tumor versursacht im frühen Stadium kaum Beschwerden, deshalb wird er meist erst in einem fortgeschritteneren Stadium diagnostiziert. Laut Prognosen des Robert Koch-Instituts sind im Jahr 2020 1.800 Frauen und 6.100 Männer neu an Speiseröhrenkrebs erkrankt. Das mittlere Erkrankungsalter bei Frauen beträgt 71 Jahre, bei Männern 67 Jahre. Das Ösophaguskarzinom zählt zu den Krebserkrankungen mit schlechter Prognose. Die relative 5-Jahres Überlebensrate liegt bei 22 bis 24 Prozent.

Die Leitlinie ist auf dieser Webseite abrufbar: https://www.leitlinienprogramm-onkologie.de/leitlinien/oesophaguskarzinom/

Zudem sind die Inhalte in der kostenfreien Leitlinien-App integriert. Nähere Informationen zur Leitlinien-App gibt es hier: https://www.leitlinienprogramm-onkologie.de/app/ 
Universitätsklinikum Schleswig-Holstein

\section{Brustkrebs-Diagnostik: Studie am UKSH weist Erfolg des QuaMaDi-Programms nach}

In Schleswig-Holstein bietet das QuaMaDiProgramm Frauen mit unklarem Tastbefund, Symptomen oder erhöhtem Brustkrebsrisiko eine qualitätsgesicherte Brustkrebsdiagnostik. QuaMaDiergänzt das bundesweit eingeführte Screeningprogramm, das auf Frauen in der Altersgruppe 50 bis 69 beschränkt ist. Wie erfolgreich QuaMaDi ist, hat nun eine umfassende Datenauswertung am Universitätsklinikum Schleswig-Holstein, Campus Kiel, gezeigt.

Brustkrebs ist die Tumorerkrankung, dieweltweit am häufigsten diagnostiziert wird. Um Tumoren möglichst früh zu erkennen, wurde in Deutschland ein Screeningprogramm für Frauen zwischen 50 und 69 Jahren eingerichtet. Darüber hinaus ermöglicht nur in Schleswig-Holstein das QuaMaDi-Programm («Qualitätsgesicherte Mammadiagnostik») Frauen auch außerhalb dieser Altersgruppe, die Symptome oder ein erhöhtes Brustkrebsrisiko aufweisen, Zugang zu einer Qualitätsdiagnostik, die weit über dieStandarduntersuchung hinausgeht. Am Universitätsklinikum Schleswig-Holstein (UKSH), Campus
Kiel, wurden nun erstmals Befunde von Frauen, die im Rahmen des QuaMaDi-Programms erhoben wurden, systematisch aufgearbeitet. «Unsere umfassende Auswertung konnte den Erfolg dieses Programms eindeutig bestätigen», sagt Prof. Dr. Christoph Röcken, Direktor des Instituts für Pathologie des UKSH und der Christian-Albrechts-Universität zu Kiel.

In Zusammenarbeit mit dem Brustzentrum und der Klinik für Gynäkologie und Geburtshilfe des UKSH, Campus Kiel, hat das Institut für Pathologie Begutachtungsberichte von 6.845 Frauen analysiert, die im Rahmen von QuaMaDi in Kiel unter anderem mittels einer Mammographie, einer Röntgenbildgebung der Brust, untersucht wurden. Die Analyse zeigte, dass durch die hohen Qualitätsstandards des Programms Brustkrebs in einem frühen und potenziell heilbaren Stadium der Erkrankung diagnostiziertwurde. Zudem konnten Risikogruppen identifiziert werden, die nicht in das deutschlandweite Screening-Programm eingeschlossen werden.
In Fällen, in denen operiert werden musste, wurden außerdem die Gewebeproben aus Biopsien, die bei Krebsverdacht zunächst durchgeführt werden, mit jenem Gewebe verglichen, das anschließend bei der Operation entfernt wurde. Der Vergleich zeigte große Übereinstimmung und bestätigte die hohe Qualität und Aussagekraft der Biopsien. «QuaMaDi trägt erheblich zur qualitätsgesicherten Diagnostik bei und ergänzt das bundesweite Mammographie-Screening-Programm», folgerte die Arbeitsgruppe.

Seit 2005 können gesetzlich versicherte Frauen mit Wohnsitz in Schleswig-Holstein am QuaMaDi-Programm teilnehmen, das sich an den Qualitätsstandards nationaler und internationaler Leitlinien orientiert. Zum Programm gehört eine unabhängige Zweitbefundung aller durchgeführten Mammographieaufnahmen und - falls notwendig eine unabhängige Drittbefundung und $\mathrm{Ab}$ klärungsdiagnostik in einem der Referenzzentren.

\section{Universität Duisburg-Essen}

\section{Essener Krebsforschung wird aus NRW-Rückkehrprogramm gefördert}

Mit dem NRW-Rückkehrprogramm holt das Landesministerium für Kultur und Wissenschaft (MKW) jedes Jahr exzellente Wissenschaftstalente aus dem Ausland nach Nordrhein-Westfalen. Mit Prof. Dr. Dr. Alpaslan Tasdogan kommt zum ersten Mal ein Wissenschaftler auf diesem Weg an die Medizinische Fakultät der Universität Duisburg-Essen (UDE). Damit verbunden ist eine Förderung des Landes von 1,25 Millionen Euro über die nächsten 5 Jahre zum Aufbau einer Nachwuchsgruppe an der Universitätsmedizin Essen.

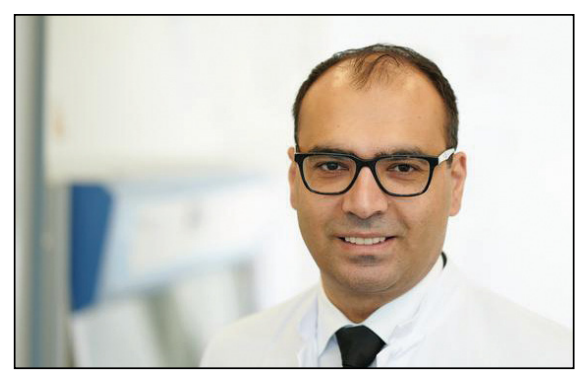

Prof. Dr. Dr. Alpaslan Tasdogan (Foto: UDE/Frank Preuß)
Prof. Dr. Dr. Tasdogan ist promovierter Onkologe und Immunologe. Er zählt zu den forschenden Ärzten und verstärkt am Universitätsklinikum Essen als Hautkrebsexperte das Team der Klinik und Poliklinik für Dermatologie, Allergologie und Venerologie. Als Professor für Tumormetabolismus an der Medizinischen Fakultät der UDE wird der 37-Jährige mit seinem Team untersuchen, wie sich normale Zellen in Krebszellen umwandeln und wo der Tumoren-Stoffwechsel (Metabolom) unter Umständen angreifbar ist.

«lch hoffe, dass wir die metabolischen Anpassungen während des komplexen Metastasierungsprozesses besser verstehen und sie auch therapeutisch nutzen können», sagt Prof. Tasdogan. Zudem wird er aktuelle Erkenntnisse in den Klinikalltag und die Behandlung von Patient:innen integrieren. «lch finde es großartig, dass sich Professor Tasdogan für uns entschieden hat», freut sich Dr. Jan Buer, Dekan der Medizinischen
Fakultät der UDE. «Seine Melanom-Expertise ist eine Bereicherung für unseren Onkologie-Schwerpunkt.»

Bevor er nach Essen kam, arbeitete er im US-amerikanischen Dallas am Children's Research Institute des Southwestern Medical Center der University of Texas. «lch hatte mich damals bewusst für Dallas entschieden», sagt Tasdogan. «Denn so konnte ich mit Professor Sean Morrison zusammenarbeiten - einer Koryphäe auf dem Gebiet der Krebsforschung, eines meiner bevorzugten Forschungsfelder.»In diesem Bereich wird der Hautkrebsexperte auch an der Universitätsmedizin Essen forschen: «Mit einem der führenden Melanom-Experten Professor Schadendorf und seinem exzellenten Team kann ich in Essen meine Forschung optimal vorantreiben», sagt Tasdogan, der nun auch Teil des Deutschen Konsortiums für Translationale Krebsforschung (DKTK) am Partnerstandort Essen/Düsseldorf ist. 


\section{Zukunftscluster-Finalist curATime: Gemeinsame Bekämpfung von Herz-Kreislauf-Erkrankungen}

Mit dem Auftakt der Konzeptionsphase des «Clusters für Atherothrombose und Individualisierte Medizin - curATime» ist jetzt auch der Startschuss für die Einbindung weiterer Netzwerkpartner aus Wissenschaft und Wirtschaft in der Rhein-MainPfalz-Region gefallen. Ziel von curATime ist es, maßgeschneiderte, innovative Behandlungsmethoden, Diagnostika und Präventionskonzepte für Herz-Kreislauf-Erkrankungen zu entwickeln. Das Cluster curATime ist einer der 15 Finalisten der «Clusters4Future»-Initiative des Bundesministeriums für Bildung und Forschung (BMBF) und ein wichtiger Baustein für den Biotechnologie-Standort Rheinland-Pfalz. Zu den Kernpartnern zählen das translationale Forschungsinstitut TRON gGmbH, die Universitätsmedizin Mainz und das Deutsche Forschungszentrum für Künstliche Intelligenz (DFKI).

«Das Cluster curATime baut auf einer vor 15 Jahren begonnenen gezielten Strukturbildung sowie umfänglichen Investitionen im Bereich der Lebenswissenschaften am Standort Rheinland-Pfalz auf. Die geplante enge Verzahnung exzellenter Grundlagenforschung mit der Nutzung eben dieser Forschungsergebnisse sowohl im klinischen als auch im ökonomischen Sinn ist ein zentrales Element des BiotechnologieProjekts der Landesregierung RheinlandPfalz. Somit ist der Erfolg des Zukunftsclusters von großer Bedeutung - für das Land Rheinland-Pfalz und auch über die Landesgrenze hinaus», betont Univ.-Prof. Dr. Georg Krausch, Präsident der Johannes Gutenberg-Universität Mainz (JGU) und Biotechnologiekoordinator des Landes RheinlandPfalz.

Mit TRON, dem Centrum für Thrombose und Hämostase (CTH) und dem Zentrum für Kardiologie an der Universitätsmedizin Mainz sowie dem DFKI haben sich im Rahmen von curATime Expert:innen auf den Gebieten der immunologischen Thromboseforschung, der Epidemiologie und Systemmedizin, der klinischen Kardiologie, der Biomarkerforschung, der Immuntherapie sowie der Künstlichen Intelligenz zusammengefunden. Die drei Kernpartner verfügen mit ihren komplementären Expertisen sowie dem Zugang zu umfangreichen Daten- und Biobanken aus großen Kohortenstudien, wie beispielsweise der GutenbergGesundheitsstudie (GHS), über ausgezeichnete Voraussetzungen, um die Clusterziele zu erreichen. Zu den Initiatoren von curATime gehören Dr. Andrée Rothermel (TRON gGmbH), Univ.-Prof. Dr. Ugur Sahin (Univer- sitätsmedizin Mainz / TRON gGmbH), Univ.Prof. Dr. Wolfram Ruf (Universitätsmedizin Mainz), Univ.-Prof. Dr. Philipp Wild (Universitätsmedizin Mainz) und Univ.-Prof. Dr. Prof. h.c. Andreas Dengel (DFKI / TU Kaiserslautern).

curATime strebt eine gesamtgesellschaftliche Vernetzung über die Wissenschaft hinaus an. Aus diesem Grund ist das Cluster auf der Suche nach weiteren Netzwerkpartnern, die sich über Forschungs- und Entwicklungsprojekte sowie innovationsbegleitende Aktivitäten an der Clusterinitiative beteiligen wollen. «curATime lebt eine offene Innovationskultur, in der wir disziplin- und branchenübergreifend zusammenarbeiten wollen, um innovative Konzepte zu entwickeln und zur Anwendung zu bringen», erläutert Dr. Andrée Rothermel, Sprecher des curATime-Clusters und Wissenschaftlicher Geschäftsführer des TRON.

Zum Ende der Konzeptionsphase entscheidet ein hochrangiges Expertengremium voraussichtlich Mitte 2022 über die weitere Förderung zwecks Realisation der Projekte des curATime-Clusters. In Aussicht steht eine Förderung in Höhe von bis zu 45 Millionen Euro für maximal drei aufeinanderfolgende jeweils dreijährige Umsetzungsphasen.

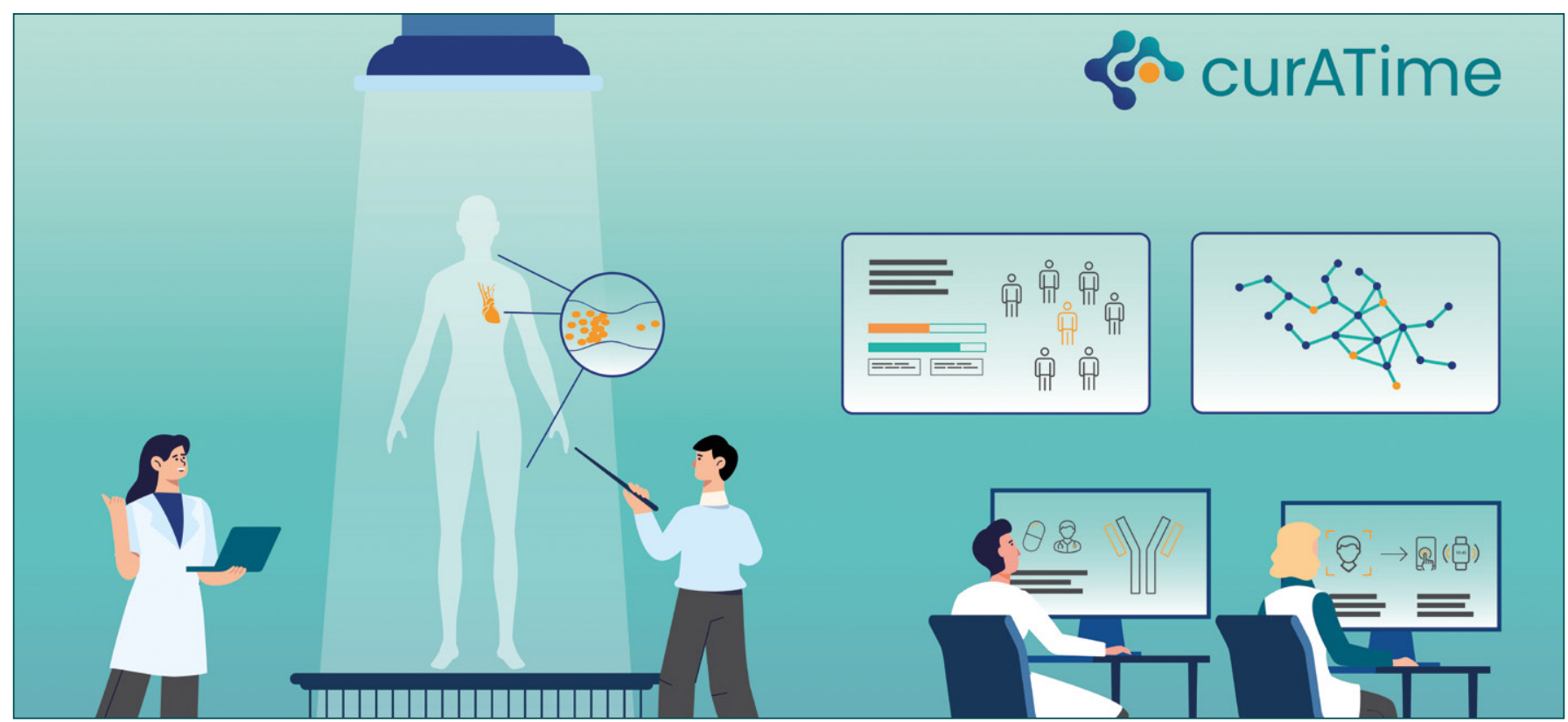

Ziel des Clusters curATime ist, durch den Einsatz von KI-Methoden in der Biomarkerforschung und der Kombination mit immunologischen Verfahren neue maßgeschneiderte Behandlungsmethoden und Präventionskonzepte für Herz- und Gefäßerkrankungen zu entwickeln. Foto: TRON gGmbH 